International Journal of Agriculture, Environment and Bioresearch

Vol. 4, No. 06; 2019

ISSN: $2456-8643$

\title{
IMPACTS OF URBAN GREENING INDUCED-SPECIES HOMOGENIZATION ON ECOSYSTEM FUNCTIONS AND SERVICES IN SOME SELECTED CAPITAL CITIES IN THE SOUTH SOUTH REGION OF NIGERIA
}

\author{
*Ita-Nya E.P. ${ }^{1}$; Obafemi A. A. ${ }^{1,2}$ and Ndukwu, B. C. ${ }^{1,3}$ \\ ${ }^{1}$ Institute of Natural Resources Environment and Sustainable Development, Faculty of Science, University of Port \\ Harcourt, Port Harcourt, Rivers State, Nigeria \\ ${ }^{2}$ Department of Geography and Environmental Management, Faculty of Social Sciences, University of Port \\ Harcourt, Port Harcourt, Rivers State, Nigeria \\ ${ }^{3}$ Department of Plant Science and Biotechnology, Faculty of Science, University of Port Harcourt, Port Harcourt, \\ Rivers State, Nigeria
}

http://doi.org/10.35410/IJAEB.2019.4478

\begin{abstract}
The study examined the impacts of urban greening induced species homogenisation on ecosystem functions and services in some selected capital cities in the South south region of Nigeria. The study established quadrats of $30 \mathrm{mx} 200 \mathrm{~m}$ along road (transects) in GRAs of Uyo City, Akwa Ibom State and Yenagoa City, Bayelsa State labelled as sampled sites and a quadrat of $100 \mathrm{mx} 100 \mathrm{~m}$ were established as control sites (secondary forest) at a minimum of $300 \mathrm{~m}$ from the sampled sites. Jaccard Similarity Index was used for the degree of homogenisation of plants while Levene Statistics was used to determine the significant differences in the degree of plant homogenisation between sample and control sites at $\mathrm{p}<0.05$ significance levels. Findings showed that Vossia cuspidata was highest $(51.4 \%)$ in one of the sampled sites in Uyo City while Cynodon dactylon highest $(80.6 \%)$ in abundance in Yenagoa in one of the sampled roads. Similarity index for homogenization was $1.6 \%$ and $10 \%$ in Uyo City and Yenagoa City respectively. Furthermore, $96.8 \%$ of identified plants were missing in Uyo (GRA) sampled sites (roads) while $84.6 \%$ of native plant species identified in the control sites are missing from the urban sampled sites in Yenagoa City. The Levene statistics have shown that there is a significant difference in the level of homogenization between sampled sites and control sites in the study area $(\mathrm{F}=34.857 ; \mathrm{p}<0.05)$. The plants in the control sites provided all ecological functions and services more than the sampled site except ornamental functions $(11.0 \%)$. The study concluded that the introduction of non-native plants as a result of urban greening activities have altered ecosystem functions and services and have promoted species homogenization in the urban centres. Based on findings, the study recommended that urban greening projects and activities should be managed effectively by the city authorities, to reduce its impacts on the plants species diversity and to improve the vegetation status which can promote ecosystem functions and services.
\end{abstract}

Keywords: Urban, Greening, Homogenization, Ecosystem functions, South-south region, Nigeria.

\section{INTRODUCTION}


Plant biodiversity is an important component of urban ecosystems, and contributes to the value of public life by enhancing air quality and improving aesthetic value of the environment in parks and recreation. However, due to urbanization, there is a tremendous and severe erosion of local plant species diversity. Urbanization generates enormous environmental changes (Zhao, et al., 2006; Pearce et al., 2018) and promotes loss of indigenous species, natural habitats and consistently reduces the accessible areas for many wild species. All these factors combined produced loss in biodiversity in an urban setting. Urban areas influences regional flora by changing the availability or spatial arrangement of habitats, their species and evolutionary selection of plants populations (Williams et al., 2010). Urban landscaping including planting of species normally supports the introduction of alien species by humans; and poses serious threat to biodiversity (Bigirimama et al., 2011). Alarmingly, growing urban landscaping is expected to promote the introduction of exotic species and this mostly leads to the extirpation of some native plants (Duncan et al., 2011), and decline of native biodiversity. Thus, accelerating urbanization and its associated processes usually lead to the introduction of alien plant species and tremendous environmental modifications favouring biotic homogenization (Abadie et al., 2011). In an urban setting, biotic homogenization was observed as an expansion of alien plant species and a reduction of native plant species (Arevalo et al., 2010).

Similarly in Nigeria, rapid urbanization and land use change in most cities has led to the alteration of structure and composition of forests (Wear, 2013). Novel ecosystem assemblages have developed in both urban and peri-urban forests in response to land use change, as well as species introductions, ecological disturbance, and socio-political and economic shifts (Conway and Bourne, 2013; Raupp et al., 2006). In addition, many urban floras include both human-cultivated and spontaneously occurring species, each of which is subject to distinct ecological and human influences (Knapp et al. 2012). Although there is evidence of high biodiversity within cities (McKinney 2006, Grimm et al., 2008; Knapp et al. 2008, Newbold et al., 2015), few studies have disentangled these ecological and human influences that drive urban biodiversity.

Although, urbanization results in native habitat destruction and is regarded as a major threat to biodiversity; some cities are richer than others in terms of plant species, both from intentional and unintentional introductions but also due to natural factors. For instance, in some cases, cities are built up in areas of natural heterogeneity which supports natural biodiversity and thus, makes it richer in terms of plant species diversity (Luc and Emmanuel, 2014).

Urban plant communities and species homogenization arise from the ecological assembly processes that operate in natural ecosystems e.g., habitat filtering (Mayfield et al., 2005; Kraft et al., 2015); and species interactions (Hillerislambers et al., 2012), as well as human desires and influences (Aronson et al., 2016; Jenerette et al., 2016). Human preferences for the species in urban greening have greatly facilitated the spread of non-native species, resulting in the homogenization of urban plant communities across spatial scales (Qians et al., 2016). Cities homogenize the physical environment because they are built to meet the relatively narrow needs of just one species, our own. Also, cities are maintained for centuries in a disequilibrium state from the local natural environment by the importation of vast resources of energy and materials. Consequently, as cities expand across the planet, biological homogenization increases because the same "urban-adaptable" species become increasingly widespread and locally abundant in cities across the planet (McKinneey, 2006). As urbanization often produces a local gradient of 
disturbance, one can also observe a gradient of homogenization. Synanthropic (human mediated symbiosis) species adapted to intensely modified built habitats at the urban core are "global homogenizers", found in cities worldwide.

Thus, there are several contrasting processes that can be observed, which are introduction of often ubiquitous alien plant species (biological invasion), decline and extirpation of native species, and a remaining high species richness of native species, based on those that are common (McKinneey, 2006; Ecological Society of America (ESA), 2016). This knowledge gap makes it difficult to interpret comparisons of diversity, both among different urban assemblages and when comparing urban and natural assemblages. As natural landscapes are altered by urbanization, there is a gap in our understanding of the implications these changes might have on regional urban and peri-urban plants diversity. Thus, advancing toward urban sustainability in the 21st century will require understanding the extent to which urbanization may be producing socially and environmentally homogeneous or differentiated landscapes at neighbourhood, metropolitan, national, and continental scales which also have several implications on loss in plants species richness and diversity. It is thus sequel to this background that the present study is focussing on the impacts of urban greening induced species homogenization on ecosystem functions and services in some selected capital cities of South south region of Nigeria.

\section{MATERIALS AND METHODS}

\section{Study Area Description}

The study was carried out in Uyo, Akwa Ibom and Yenagoa, Bayelsa States in the South south region of Nigeria (Figure 1). The South south region is found within the Niger Delta of Nigeria. South south region of Nigeria is located between latitudes $5^{\circ} 00^{\prime} \mathrm{N}$ and $6^{\circ} 30^{\prime} \mathrm{N}$ and longitudes $5^{\circ}$ $20^{\prime} \mathrm{E}$ and $9^{\circ} 00^{\prime} \mathrm{E}$. The South-south region with the Niger River is sitting directly on the Gulf of Guinea on the Atlantic Ocean in Nigeria. The study area features a tropical monsoon climate, designated by the Koppen climate classification as "Am", and it is mostly found in the southern part of the country. This climate is influenced by the monsoons originating from the South Atlantic Ocean, which is brought into the country by the maritime tropical air mass, a warm moist sea to land seasonal wind (Britanica, 2014). Its warmth and high humidity gives it a strong tendency to ascend and produce copious rainfall, which is a result of the condensation of water vapour in the rapidly rising air (Park, 2004). The temperature ranges are almost constant throughout the year. The South-south region of Nigeria experiences heavy and abundant rainfall. These storms are usually conventional in nature due to the regions proximity, to the equatorial belt. The annual rainfall received in this region is very high, usually above the $2,000 \mathrm{~mm}$ (78.7 in) rainfall totals giving for tropical rainforest climates worldwide. Over $4,000 \mathrm{~mm}$ of rainfall is received in the coastal region of Nigeria around the Niger Delta area. Bonny town found in the coastal region of the Niger delta area in southern Nigeria receives well over 4,000 $\mathrm{mm}$ of rainfall annually (Geographical Alliance of Iowa, 2010). The geology includes a new threefold lithostratigraphic subdivision comprising an upper sandy Benin formation, an intervening unit of alternating sandstone and shale named the Agbada formation, and a lower shaly Akata formation. These three units extend across the whole delta and each ranges in age from early Tertiary to Recent (Short and Staeuble, 1967). The south-south region is well drained with both fresh and salt water. The salt water is caused by the intrusion of seawater inland, thereby making the water slightly salty. Drainage of the study area is poor because of the 
presence of many surface water and heavy rainfall between $2000 \mathrm{~mm}$ and $2400 \mathrm{~mm}$ (Mmom and Fred-Nwagwu, 2013). The vegetation includes the rainforest, swampy forest and mangrove (Geographical alliance of Iowa, 2010). The primary economic activities in most rural communities in the south-south region include peasant farming, petty trading and fishing, shifting cultivation (Slash and burn), which involves cultivating a piece of land for a number of years and then abandoning it for a more fertile land is traditionally practised in the area. Some of the cash crops grown in the study area include oil palm (Elaeis guineensis), cacao (Theobroma cacao), cassava (Manihot esculenta) and rubber (Herea brasiliensis) (Enaruvbe and Atafo, 2015).

\section{Plant Species Identification and Enumeration}

The vegetation makes up of sampled roads in each major urban centre's government residential areas (GRAs) and control sites (Table 1). The study made use of (3) major street roads in the GRAs in each major cities, whereby plants were identified and enumerated in order to understand their vegetation status. These roads were selected based on their high vegetation composition and status, while the control sites were selected based on the diverse diverse species of plants can be enumerated and used as basis of comparison for the research. The control sites are the primary or secondary forest, nature parks or any other relatively undisturbed forests in each study area. The control sites were located at a minimum of $300 \mathrm{~m}$ away from the sampled roads (sites). The study applied transect methods whereby quadrats of $30 \mathrm{~m}$ by $200 \mathrm{~m}$ used for the data collection were selected within each transect (street road). In other words several quadrats were established regularly in relation to the road length for each sampled street roads. Therefore, plant types were identified and enumerated on the spot with the help of a Taxonomist from the start to the end of the street road (transect). Quadrats of $30 \mathrm{~m} \times 200 \mathrm{~m}$ were laid on both sides of the road and a gap of $100 \mathrm{~m}$ was created till the next quadrat and so on until the end of the street road (Figure 2). On the other hand, the control sites plant species were identified within selected secondary forest using also quadrat methods. Five (5) $30 \mathrm{~m}$ x $30 \mathrm{~m}$ randomly selected quadrats were delimited within quadrats of $100 \mathrm{~m} \times 100 \mathrm{~m}$ laid within each control sites for the collection of data on the vegetal composition and the plant species types. The data collection exercise was carried out between March and June, 2019 (for a period of four (4) months). The data collected on plant types and composition were used for the computation of analytical vegetation features such as species composition, species density, species diversity, species richness, species evenness and similarity index which followed standard phyto-sociological methods. The identification of plant was also carried out with the help of a Taxonomist from the University of Port Harcourt. The plants that were not identified in situ were taken to the Herbarium in the University for Proper Identification.

Species composition of plants were determined by identifying the plant species while the population of individual species were determined by direct counting of the population of each species in the sampled roads and control sites. Plant species similarity index using Jaccard Index was used to ascertain the degree of plant species homogenization missing from urban greening activities in the study area. The impact of urban greening induced species homogenization on ecosystem functions and services were discussed based on the use of checklist of the plants. Both descriptive and inferential statistics were used for the data analysis. Levene Statistics Homogeneity of variance was used to determine the difference in the number of plant species 
identified and level of homogenization among sampled sites and control sites in the study area. The analysis was computed using SPSS version 24.0.

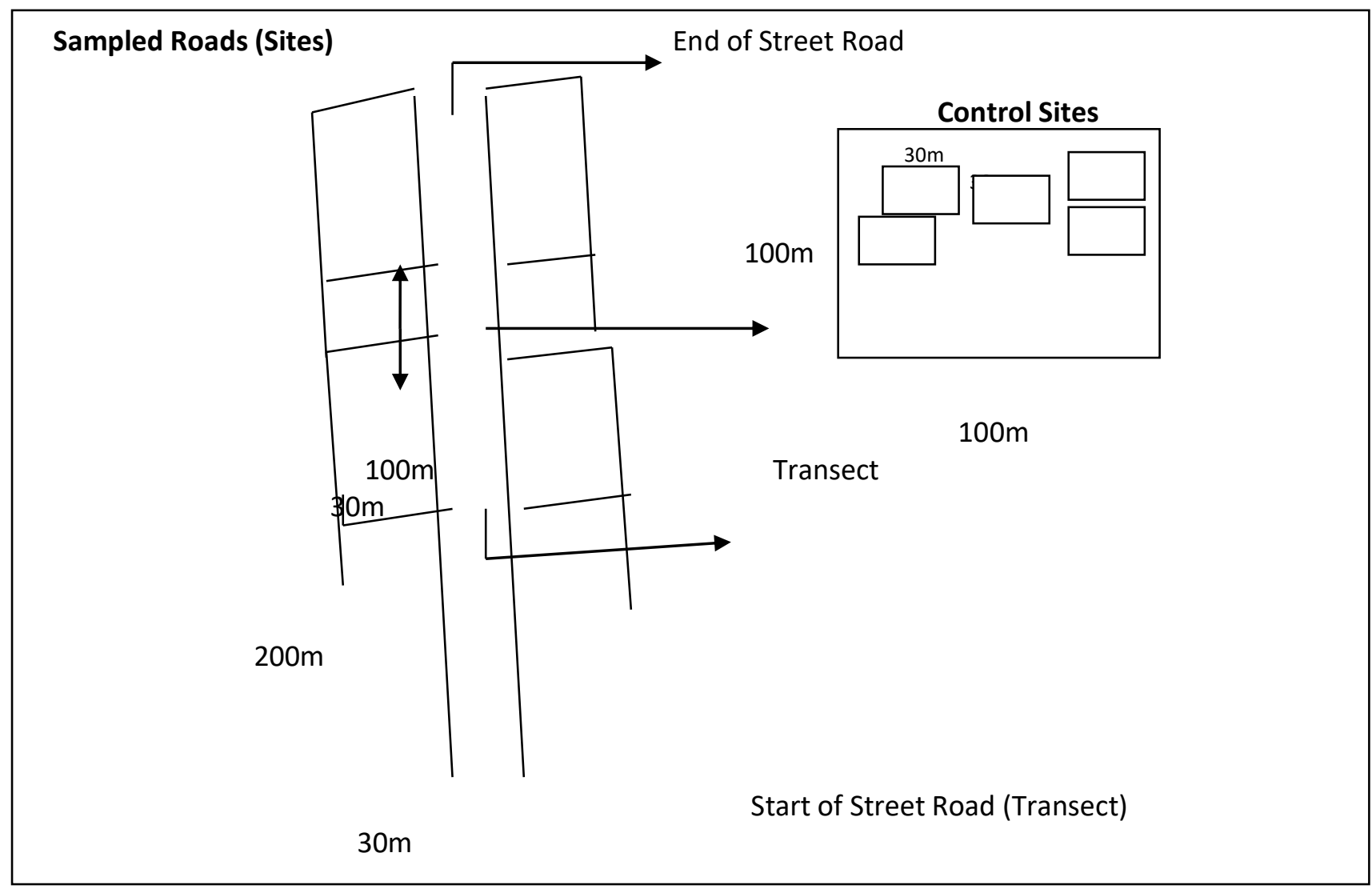

Figure 2: Method of collection of plant species types and composition in the study area Table 1: Study Areas/Sampled Streets/Roads Names and Locations

\begin{tabular}{|l|l|l|l|l|l|}
\hline State & Capital & GRA & \multirow{2}{*}{$\begin{array}{l}\text { Selected Street } \\
\text { name/Sampled Sites }\end{array}$} & \multicolumn{2}{|c|}{ Location } \\
\cline { 4 - 6 } & Cities & & Northings & Eastings \\
\hline \multirow{3}{*}{ Akwa Ibom } & \multirow{2}{*}{ Uyo } & Ewet Housing & Godwin Abe/1 & $5.01188^{0}$ & $7.95012^{0}$ \\
\cline { 3 - 6 } & & & G-Lane/2 & $5.01677^{0}$ & $7.94520^{0}$ \\
\cline { 3 - 6 } & & Lagos Street/3 & $5.01281^{0}$ & $7.94528^{0}$ \\
\hline \multirow{2}{*}{ Bayelsa } & \multirow{2}{*}{ Yenagoa } & Otitio GRA & Biogbolo/1 & $4.93921^{0}$ & $6.32203^{0}$ \\
\cline { 3 - 6 } & & Erepa/2 & $4.93361^{0}$ & $6.32187^{0}$ \\
\cline { 3 - 6 } & & Otitio/3 & $4.93638^{0}$ & $6.31922^{0}$ \\
\hline Control Sites & & & $5.05422^{0}$ & $7.92774^{0}$ \\
\hline Akwa Ibom & \multicolumn{2}{|l|}{ Uyo (Secondary Forest) } & $5.14036^{0}$ & $6.44856^{0}$ \\
\hline Bayelsa & \multicolumn{2}{|l|}{ Yenagoa (Okordia clan secondary forest) } &
\end{tabular}


Vol. 4, No. 06; 2019

ISSN: $2456-8643$

\section{RESULTS AND DISCUSSIONS}

\section{Plant Species Identified along Sampled Roads across the Selected Cities}

In Uyo, the information for the plant species types identified sampled and control sites are displayed on Table 2 and Figure 3. In the first sampled site, it was displayed that Caesalpina pulcherrima recorded $4.7 \%$ individuals, Carica papaya recorded $8.2 \%$ individuals, Cocos nucifera recorded $8.2 \%$ individuals, Cuphea California Torr recorded 3.5\% individuals, Ficus benjamina L. and Nutt. recorded $4.7 \%$ and $5.9 \%$ individuals respectively; Hibiscus arnottiamus recorded 2.4\% individuals, Hura crepitans was 3.5\% individuals, Mangifera indica was 12.9\% individuals, Musa sapientum was $10.6 \%$ individuals, Nerium oleander L. recorded $4.7 \%$ individuals, Polyalthia longifolia recorded $15.3 \%$ individuals, Psidium guajava recorded $7.1 \%$ individuals, Syagrus romanzoffiana also recorded $4.7 \%$ individuals, while Terminalia mantalis recorded 3.5\% individuals from total percentage of identified plants. The study discovered that Polyalthia longifolia (15.3\%) recorded the highest number of individual plant species. A total of 15 individuals of different plant species with an overall total number of 85 individual plant compositions were identified.

In second sampled site, it was revealed that Carica papaya recorded $4.0 \%$ individuals, Delonix regia recorded $4.0 \%$ individuals, Ficus nitida recorded 2.3\% individuals, Hibiscus arnottiamus recorded 2.8\% individuals, Musa paradisiacal recorded 5.2\% individuals, Musa sapientum was $6.9 \%$ individuals, Polyalthia longifolia recorded $10.4 \%$ individuals, Psidium guajava recorded $7.5 \%$ individuals, Rhizophora mangus recorded $1.7 \%$ individuals, Terminalia cattapa recorded $3.5 \%$ individuals, while the remaining percentage from the total of identified plant species was Vossia cuspidata and it recorded 51.4\% number of individuals in the study area. For sampled trees and shrubs, Psidium guajava recorded the highest while only one type of grass (herbs) was identified (Vossia cuspidata). A total of 11 individuals of different plant species with an overall total of 173 individual plant compositions were identified.

In the third sampled site displayed in Table 6, Albizia zygia recorded 3.0\% individuals, Anacardium occidentale recorded $4.5 \%$ individuals, Anona nuricata recorded $4.5 \%$ individuals, Carica papaya recorded $11.9 \%$ individuals, Citrus spp recorded $9.0 \%$ individuals, Cocos nucifera recorded 9.0\% individuals, Cycas revolute recorded 6.0\% individuals, Elaeis guineensis recorded 9.0\% individuals, Erythrophlem ivorensis recorded 6.0\% individuals, Ficus benjamina recorded 7.5\% individuals, Ficus carica recorded 6.0\% individuals, Mangifera indica recorded 9.0\% individuals, Persea Americana recorded 3.0\% individuals, Ralphia hookeri recorded 9.0\% individuals, while Spondiae cythera recorded 3.0\% individuals in the study area. An overall total of 67 individuals of plant species from 15 individuals of different species were identified in the third sampled site.

In the control site, the plant composition revealed that A.laxiflora recorded $1.9 \%$ individuals, Acioa barteri recorded $1.0 \%$ individuals, Albizia adianthifolia recorded $1.0 \%$ individuals, Alstonia boonei recorded 2.2\% individuals, Anacardium occidentale recorded 1.0\% individuals, Anthocleisti vogelii recorded $1.3 \%$ individuals, Anthonotha macrophylla recorded $0.6 \%$ individuals, Antiaris Africana recorded 1.0\% individuals, Bambusa vulgaris recorded 1.3\% individuals, Baphia nitida recorded 1.6\% individuals, Bombax buonopozense recorded $0.6 \%$ individuals, Centrosema pubescens recorded $13.5 \%$ individuals, Chromolaena odorata recorded 9.0\% individuals, Cleistopholis patens recorded $7.1 \%$ individuals, Cola acuminate recorded 
$10.6 \%$ individuals, Combretum albidum recorded $8.7 \%$ individuals, Costus afer recorded $11.2 \%$ individuals, Dracena $s p$ recorded $1.3 \%$ individuals, Elaeis guineensis recorded $3.8 \%$ individuals, Ficus exasperata recorded 2.2\% individuals, Garcinia manii recorded $0.6 \%$ individuals, Harungana madagascariensis recorded $2.6 \%$ individuals, Leea guineensis recorded $1.9 \%$ individuals, Musanga cecropioides recorded 3.5\% individuals, Myrianthus arboreus recorded $2.2 \%$ individuals, Pterocarpus mildbraedii recorded 0.6\% individuals, Raphia spp recorded $1.6 \%$ individuals, Senna alata recorded $2.9 \%$ individuals, Terminalia ivorensis recorded $1.0 \%$ individuals, while Urena lobata recorded $0.6 \%$ individuals.

Table 2: Identified Plant Species in Sampled and Control Site in Uyo, Akwa Ibom State

\begin{tabular}{|c|c|c|c|}
\hline Site 1 & Plant Species Types & Frequency & $\%$ \\
\hline 1 & Caesalpinia pulcherrima & 4 & 4.7 \\
\hline 2 & Carica papaya & 7 & 8.2 \\
\hline 3 & Cocos nucifera & 7 & 8.2 \\
\hline 4 & Cuphea california Torr. & 3 & 3.5 \\
\hline 5 & Ficus benjamina $L$. & 4 & 4.7 \\
\hline 6 & Ficus benjamina Nutt. & 5 & 5.9 \\
\hline 7 & Hibiscus arnottianus & 2 & 2.4 \\
\hline 8 & Hura crepitan & 3 & 3.5 \\
\hline 9 & Mangifera indica & 11 & 12.9 \\
\hline 10 & Musa sapientum & 9 & 10.6 \\
\hline 11 & Nerium oleander $L$. & 4 & 4.7 \\
\hline 12 & Polyalthia longifolia & 13 & 15.3 \\
\hline 13 & Psidium guajava & 6 & 7.1 \\
\hline 14 & Syagrus romanzoffiana & 4 & 4.7 \\
\hline \multirow[t]{2}{*}{15} & Terminalia mantaly & 3 & 3.5 \\
\hline & Total & 85 & 100.0 \\
\hline Site 2 & Plant Species Types & Frequency & $\%$ \\
\hline 1 & Carica papaya & 7 & 4.0 \\
\hline 2 & Delonix regia & 7 & 4.0 \\
\hline 3 & Ficus nitida & 4 & 2.3 \\
\hline 4 & Hibiscus arnottians & 5 & 2.9 \\
\hline 5 & Musa parasidiaca & 9 & 5.2 \\
\hline 6 & Musa sapientum & 12 & 6.9 \\
\hline 7 & Polyalthia longifolia & 18 & 10.4 \\
\hline 8 & Psidium guajava & 13 & 7.5 \\
\hline 9 & Rhizophora mangus & 3 & 1.7 \\
\hline 10 & Terminalia cattapa & 6 & 3.5 \\
\hline \multirow[t]{2}{*}{11} & Vossia cuspidata & 89 & 51.4 \\
\hline & Total & 173 & 100.0 \\
\hline Site 3 & Species Types & Frequency & $\%$ \\
\hline
\end{tabular}


International Journal of Agriculture, Environment and Bioresearch

Vol. 4, No. 06; 2019

ISSN: $2456-8643$

\begin{tabular}{|c|c|c|c|}
\hline 1 & Albizia zygia & 2 & 3.0 \\
\hline 2 & Anacardium occidentale & 3 & 4.5 \\
\hline 3 & Anona nuricata & 3 & 4.5 \\
\hline 4 & Carica papaya & 8 & 11.9 \\
\hline 5 & Citrus spp & 6 & 9.0 \\
\hline 6 & Cocos nucifera & 6 & 9.0 \\
\hline 7 & Cycas revoluta & 4 & 6.0 \\
\hline 8 & Elaeis guineensis & 6 & 9.0 \\
\hline 9 & Erythrophlem ivorensis & 4 & 6.0 \\
\hline 10 & Ficus benjamina & 5 & 7.5 \\
\hline 11 & Ficus carica & 4 & 6.0 \\
\hline 12 & Mangifera indica & 6 & 9.0 \\
\hline 13 & Persea americana & 2 & 3.0 \\
\hline 14 & Ralphia hookeri & 6 & 9.0 \\
\hline 15 & Spondiae cythera & 2 & 3.0 \\
\hline & Total & 67 & 100.0 \\
\hline Control Site & Plant Species Types & Frequency & $\%$ \\
\hline 1 & A. laxiflora & 6 & 1.9 \\
\hline 2 & Acioa barteri & 3 & 1.0 \\
\hline 3 & Albizia adianthifolia & 3 & 1.0 \\
\hline 4 & Alchornea cordifolia & 5 & 1.6 \\
\hline 5 & Alstonia boonei & 7 & 2.2 \\
\hline 6 & Anacardum occidentalis Linn & 3 & 1.0 \\
\hline 7 & Anthocleista vogelii & 4 & 1.3 \\
\hline 8 & Anthonotha macrophylla & 2 & 0.6 \\
\hline 9 & Antiaris africana & 3 & 1.0 \\
\hline 10 & Bambusa vulgaris & 4 & 1.3 \\
\hline 11 & Baphia nitida & 5 & 1.6 \\
\hline 12 & Bombax buonopozense & 2 & 0.6 \\
\hline 13 & Centrosema pubescens & 42 & 13.5 \\
\hline 14 & Chromolaena odorata & 28 & 9.0 \\
\hline 15 & Cleistopholis patens & 22 & 7.1 \\
\hline 16 & Cola acuminate & 33 & 10.6 \\
\hline 17 & Combretum albidum & 27 & 8.7 \\
\hline 18 & Costus afer & 35 & 11.2 \\
\hline 19 & Dracena sp. Linn. & 4 & 1.3 \\
\hline 20 & Elaeis guineensis & 12 & 3.8 \\
\hline 21 & Ficus exasperata & 7 & 2.2 \\
\hline 22 & Garcinia manii & 2 & 0.6 \\
\hline 23 & Harungana madagascariensis & 8 & 2.6 \\
\hline
\end{tabular}


International Journal of Agriculture, Environment and Bioresearch

Vol. 4, No. 06; 2019

ISSN: $2456-8643$

\begin{tabular}{|c|l|c|c|}
\hline 24 & Leea guineensis & 6 & 1.9 \\
\hline 25 & Musanga cecropioides & 11 & 3.5 \\
\hline 26 & Myrianthus arboreus & 7 & 2.2 \\
\hline 27 & Pterocarpus mildbraedii & 2 & 0.6 \\
\hline 28 & Raphia spp & 5 & 1.6 \\
\hline 29 & Senna alata & 9 & 2.9 \\
\hline 31 & Terminalia ivorensis & 2 & 1.0 \\
\hline 32 & Urena lobata Total & 312 & 0.6 \\
\hline & \multicolumn{1}{|c|}{ Ton } \\
\hline
\end{tabular}

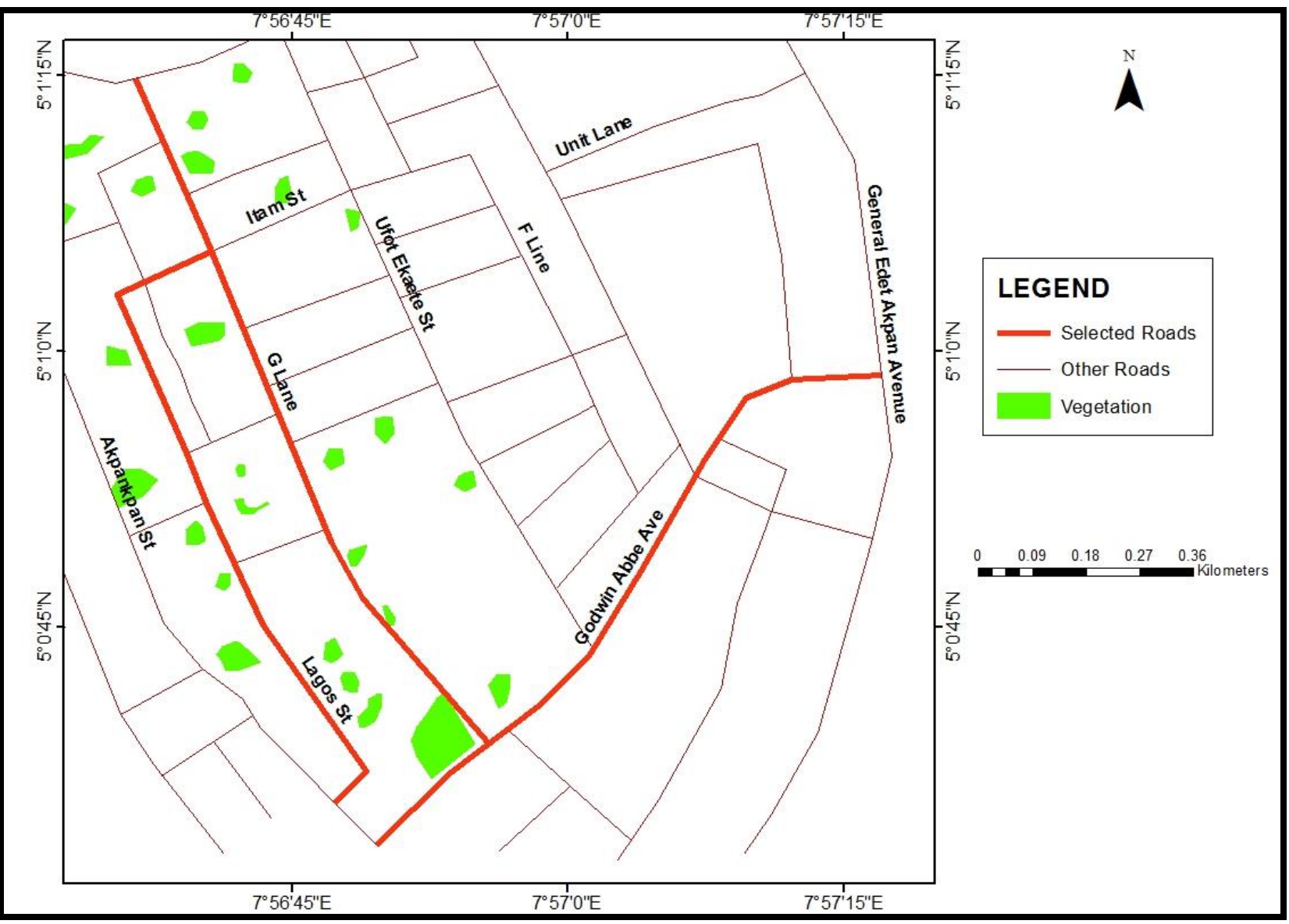

Figure 3: Selected Sampled Sites (Roads) in Ewet Housing Estate (GRA), Uyo, Akwa Ibom

In Yenagoa, the plant compositions in the selected road samples and control site are displayed in Table 3 and Figure 4. In sample site 1, the distribution revealed that Carica papaya recorded $3.7 \%$ individuals, Cocos nucifera recorded 3.7\% individuals, Cycas cecenalis recorded 1.7\% individuals, Cynodon dactylon recorded $80.6 \%$ individuals, Delonix regia recorded $0.9 \%$ individuals, Mangifera indica recorded $2.3 \%$ individuals, Musa sapientum was $1.1 \%$ individuals, 
Polyalthia longifolia recorded $2.3 \%$ individuals, Psidium guajava recorded $2.9 \%$ individuals, while Thuja sinensis recorded $0.9 \%$ individuals from total percentage of identified plants. A total of 10 individuals of different plant species with an overall total number of 350 individual plant compositions were identified.

The information for the plant species types identified under sampled sites 2 revealed that Carica papaya recorded $4.0 \%$ individuals, Delonix regia recorded $4.0 \%$ individuals, Mangifera indica recorded $8.0 \%$ individuals, Musa paradisiaca recorded $17.3 \%$ individuals, Polyalthia longifolia recorded $24.0 \%$ individuals, Psidium guajava recorded $10.7 \%$ individuals, Spondias cethera recorded $4.0 \%$ individuals, while Terminalia mantaly recorded $16.0 \%$ individuals. A total of 9 individuals of different plant species types with an overall total of 75 individual plant compositions were identified.

In the third sampled site in Yenagoa; Alchornea cordifolia recorded $16.2 \%$ individuals, Bambusa vulgaris recorded $6.8 \%$ individuals, Carica papaya recorded $4.1 \%$ individuals, Citrus spp recorded $5.4 \%$ individuals, Delonix regia recorded $13.5 \%$ individuals, Elaeis guineensis recorded $13.5 \%$ individuals, Mangifera indica recorded $4.1 \%$ individuals, Musa paradisica recorded $8.1 \%$ individuals, Musa sapientum recorded $5.4 \%$ individuals, Psidium guajava recorded $10.8 \%$ individuals, Terminalia cattapa recorded $5.4 \%$, while Terminalia mantaly recorded $6.8 \%$ individuals in the study area. An overall total of 74 individuals of plant species from 15 different plant species types were identified in the third sampled site.

The identified plant species types recorded under the control site were showed that Alchornea cordifolia recorded $2.7 \%$ individuals, Alstonia boonei recorded $3.4 \%$ individuals, Alstonia congesis recorded $2.7 \%$ individuals, Anthocleistii vogelii recorded $2.7 \%$ individuals, Anthonotha macrophylla recorded $2.0 \%$ individuals, Bambusa vulgaris recorded $8.7 \%$ individuals, Bridella micrantha recorded $2.0 \%$ individuals, Cleistopholis patens recorded $2.0 \%$ individuals, Combretum micranthia recorded $7.4 \%$ individuals, Elaeis guineensis recorded $14.8 \%$ individuals, Endodesima calophylloides recorded 3.4\% individuals, Erasmopatha microcapa recorded 2.7\% individuals, Garcinia kola recorded 2.0\% individuals, Guarea cedrata recorded $5.3 \%$ individuals, Harungana madagascariensis recorded $2.0 \%$ individuals, Hevea brasiliensis recorded $4.7 \%$ individuals, Lophira Alata recorded 2.7\% individuals, Militia aboensis recorded $1.3 \%$ individuals, Musanga cecropioides recorded 3.4\% individuals, Newbouldia laevis recorded $1.3 \%$ individuals, Picanthus agolensis recorded $4.2 \%$ individuals, Psidium guajava recorded $6.7 \%$ individuals, Raphia manii recorded $2.7 \%$ individuals, Raphia vinifera recorded $5.4 \%$ individuals, Rauvolfia vomitoria recorded $2.7 \%$ individuals, while lastly Rhizophora racemosa recorded $1.3 \%$ individuals in the study area.

Table 3: Identified Plant Species in Sampled Sites 1, 2 and 3 in Yenagoa, Bayelsa State

\begin{tabular}{|c|l|c|c|}
\hline Site 1 & \multicolumn{1}{|c|}{ Species Types } & Frequency & \% \\
\hline 1 & Carica papaya & 13 & 3.7 \\
\hline 2 & Cocos nucifera & 13 & 3.7 \\
\hline 3 & Cycas cecenalis & 6 & 1.7 \\
\hline 4 & Cynodon dactylon & 282 & 80.6 \\
\hline
\end{tabular}


International Journal of Agriculture, Environment and Bioresearch

Vol. 4, No. 06; 2019

ISSN: $2456-8643$

\begin{tabular}{|c|c|c|c|}
\hline 5 & Delonix regia & 3 & 0.9 \\
\hline 6 & Mangifera indica & 8 & 2.3 \\
\hline 7 & Musa sapientum & 4 & 1.1 \\
\hline 8 & Polyalthia longifolia & 8 & 2.3 \\
\hline 9 & Psidium guajava & 10 & 2.9 \\
\hline \multirow[t]{2}{*}{10} & Thuja sinensis & 3 & 0.9 \\
\hline & Total & 350 & 100.0 \\
\hline Site 2 & Species Types & Frequency & $\%$ \\
\hline 1 & Carica papaya & 5 & 6.7 \\
\hline 2 & Cycas cecenalis & 7 & 9.3 \\
\hline 3 & Delonix regia & 3 & 4.0 \\
\hline 4 & Mangifera indica & 6 & 8.0 \\
\hline 5 & Musa paradisica & 13 & 17.3 \\
\hline 6 & Polyalthia longifolia & 18 & 24 \\
\hline 7 & Psidium guajava & 8 & 10.7 \\
\hline 8 & Spondias cethera & 3 & 4.0 \\
\hline \multirow[t]{2}{*}{9} & Terminalia mantaly & 12 & 16.0 \\
\hline & Total & 75 & 100.0 \\
\hline Site 3 & Species Types & Frequency & $\%$ \\
\hline 1 & Alchornea cordifolia & 12 & 16.2 \\
\hline 2 & Bambusa vulgaris & 5 & 6.8 \\
\hline 3 & Carica papaya & 3 & 4.1 \\
\hline 4 & Citrus spp & 4 & 5.4 \\
\hline 5 & Delonix regia & 10 & 13.5 \\
\hline 6 & Elaeis guineensis & 10 & 13.5 \\
\hline 7 & Mangifera indica & 3 & 4.1 \\
\hline 8 & Musa paradisica & 6 & 8.1 \\
\hline 9 & Musa sapientum & 4 & 5.4 \\
\hline 10 & Psidium guajava & 8 & 10.8 \\
\hline 11 & Terminalia cattapa & 4 & 5.4 \\
\hline \multirow[t]{2}{*}{12} & Terminalis mantaly & 5 & 6.8 \\
\hline & Total & 74 & 100.0 \\
\hline Control Site & Species Types & Frequency & $\%$ \\
\hline
\end{tabular}


International Journal of Agriculture, Environment and Bioresearch

Vol. 4, No. 06; 2019

ISSN: $2456-8643$

\begin{tabular}{|c|c|c|c|}
\hline 1 & Alchornea cordifolia & 4 & 2.7 \\
\hline 2 & Alstonia boonei & 5 & 3.4 \\
\hline 3 & Alstonia congesis & 4 & 2.7 \\
\hline 4 & Anthocleistii vogelii & 4 & 2.7 \\
\hline 5 & Anthonotha macrophylla & 3 & 2.0 \\
\hline 6 & Bambusa vulgaris & 13 & 8.7 \\
\hline 7 & Bridella micrantha & 3 & 2.0 \\
\hline 8 & Cleistopholis patens & 3 & 2.0 \\
\hline 9 & Combretum micranthia & 11 & 7.4 \\
\hline 10 & Elaeis guineensis & 22 & 14.8 \\
\hline 11 & Endodesima calophylloides & 5 & 3.4 \\
\hline 12 & Erasmopatha microcapa & 4 & 2.7 \\
\hline 13 & Garcinia kola & 3 & 2.0 \\
\hline 14 & Guarea cedrata & 8 & 5.4 \\
\hline 15 & Harungana madagascariensis & 3 & 2.0 \\
\hline 16 & Hevea brasiliensis & 7 & 4.7 \\
\hline 17 & Lophira Alata & 4 & 2.7 \\
\hline 18 & Militia aboensis & 2 & 1.3 \\
\hline 19 & Musanga cecropioides & 5 & 3.4 \\
\hline 20 & Newbouldia laevis & 2 & 1.3 \\
\hline 21 & Picanthus agolensis & 6 & 4.0 \\
\hline 22 & Psidium guajava & 10 & 6.7 \\
\hline 23 & Raphia manii & 4 & 2.7 \\
\hline 24 & Raphia vinifera & 8 & 5.4 \\
\hline 25 & Rauvolfia vomitoria & 4 & 2.7 \\
\hline 26 & Rhizophora racemosa & 2 & 1.3 \\
\hline & Total & 149 & 100.0 \\
\hline
\end{tabular}




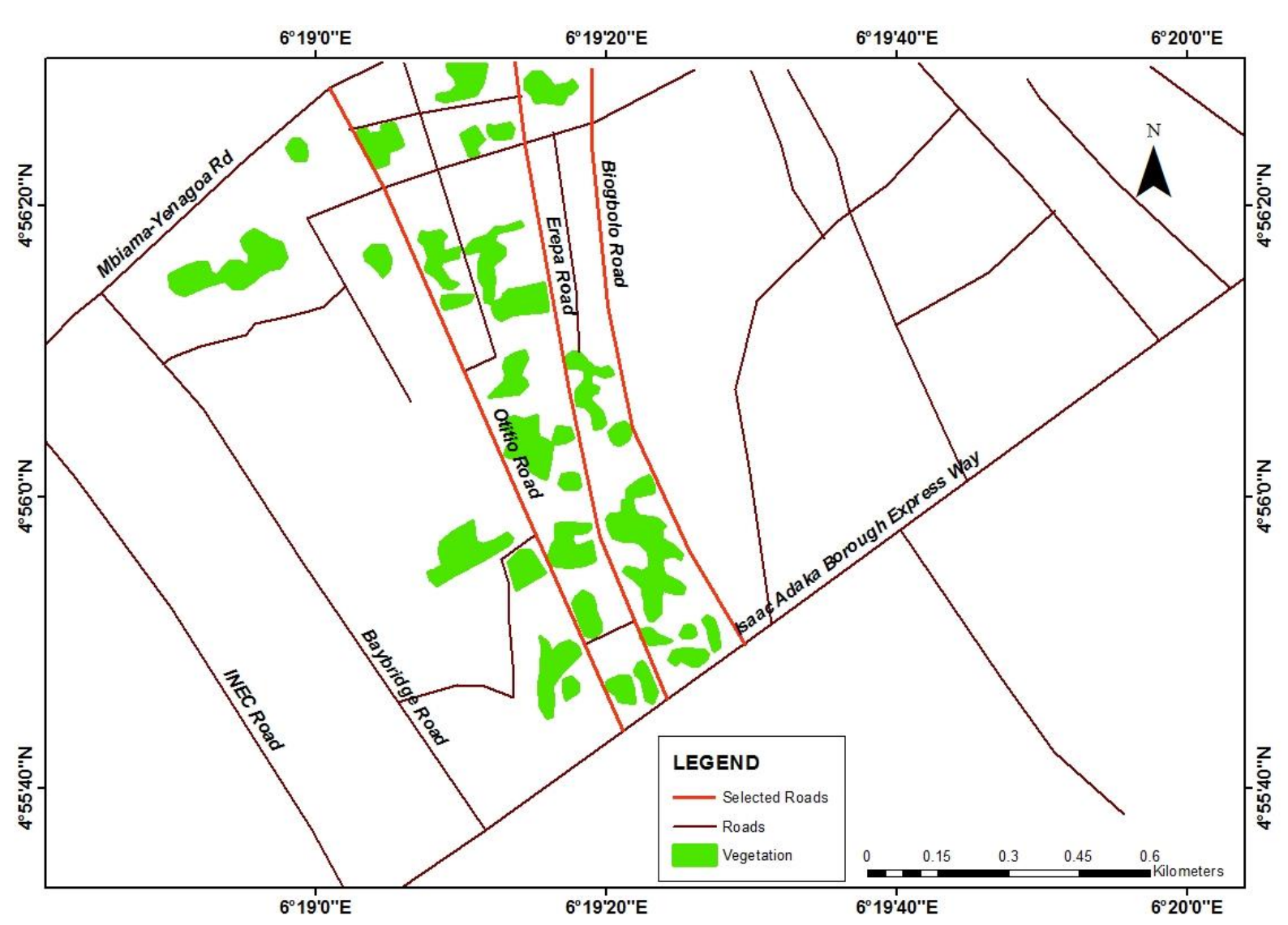

Figure 4: Selected Sample Sites (Roads) in Yenagoa, Bayelsa State

Degree of Plant Species Homogenization and Implication to Ecosystem Functions and Services

The degree of plant species homogenization in the study area was computed using the Jaccard Index of Similarity Coefficient which is a range between 0\% and 100\% (higher percentages indicate more similarity between two populations or community of species). Aside computing their similarity index, the $\mathrm{J}(\mathrm{X}, \mathrm{Y})=\mathrm{X} \cap \mathrm{Y}$ formula was used to ascertain the missing plant species as affected by urban greening activities. Thus, all sampled sites within each study site were compared with their control sites. Thereafter, their percentage compositions were determined to evaluate the degree of missing plants. The information on Tables 2 and 3 were used for this analysis. The results of the similarity index computed for all study sites displayed in Table 4 showed that the similarity index between sampled sites and control sites in Uyo study sites was $1.6 \%$ which indicated a very low index. This was because $96.9 \%$ of identified plant species in both sampled sites and control sites were non-native plant. The study discovered that a total of 30 plant species in the control sites are different from those found in the sampled sites. Thus, 96.8\% of identified plants are missing in Uyo (GRA) sampled sites (roads). In Yenagoa study site, $77.8 \%$ from the total identified plant from both sampled sites and control sites are non-native plant species. It was discovered that $84.6 \%$ of native plant species identified in the control sites are missing from the urban sampled sites. 
With the ecosystem functions and services in Table 5, the study has shown that for the erosion control and reclaiming of land, control sites recorded $2.7 \%$ plants while sampled sites recorded $2.3 \%$ plants. However, in relation to their ornamental functions, $11.0 \%$ was recorded for sampled sites against $3.2 \%$ for control sites. This means that sampled sites due to the nature of plants have more ornamental plants than identified plant species in the control sites. For their ethno medicinal values and functions, control sites recorded $11.9 \%$ plants while it showed that only $6.9 \%$ of plants under the sampled sites have these functions. The implication here is that plants grown in urban areas favours mostly non-native plant species that promotes species homogenization and most times these practices have direct impacts on plant species diversity, richness and similarity index. Therefore, it can be concluded that more diverse plant species with no disturbance will have higher ecosystem functions. Urban greening promotes the introduction of exotic plant species that have higher ornamental functions and values; of which most species introduced are invasive and could lead to habitat loss and fragmentation of native plant species. The Levene statistics have shown that there is a significant difference in the number of plant species identified and level of homogenization between sampled sites and control sites in the study area $(\mathrm{F}=34.857 ; \mathrm{p}<0.05)$.

Table 4: Similarity Index and Degree of plant species Homogenization between Sampled Sites and Control Sites in the Study Area

\begin{tabular}{|c|c|c|c|c|c|c|}
\hline $\mathrm{S} / \mathrm{N}$ & Study Sites & $\begin{array}{l}\text { Sampled Sites }(\mathrm{X}) \text { and } \\
\text { Control Sites }(\mathrm{Y})\end{array}$ & $\begin{array}{l}\text { Number } \\
\text { of } \\
\text { different } \\
\text { plants }\end{array}$ & $\begin{array}{l}\text { Total } \\
\text { no } \\
\text { missing }\end{array}$ & $\begin{array}{l}\% \text { of plant } \\
\text { species } \\
\text { homogenization }\end{array}$ & $\begin{array}{l}\% \\
\text { missing }\end{array}$ \\
\hline 1 & Uyo & $\begin{array}{c}X \cap Y=1 \\
X \text { Ủ Y }=62 \\
X \cap Y / X \text { Ü Y } * 100 \\
\mathbf{J = 1 . 6 \%}\end{array}$ & 31 & 30 & 96.9 & 96.8 \\
\hline 2 & Yenagoa & $\begin{array}{c}\mathrm{X} \cap \mathrm{Y}=4 \\
\mathrm{X} U \mathrm{U}=40 \\
\mathrm{X} \cap \mathrm{Y} / \mathrm{X} \text { Ü Y } * 100 \\
\mathbf{J}=\mathbf{1 0 \%}\end{array}$ & 14 & 22 & 77.8 & 84.6 \\
\hline
\end{tabular}


International Journal of Agriculture, Environment and Bioresearch

Vol. 4, No. 06; 2019

ISSN: $2456-8643$

Table 5: Checklist for Uses, Functions and Services of Identified plant Species in all Study Sites

\begin{tabular}{|c|c|c|c|c|c|}
\hline $\mathrm{S} / \mathrm{N}$ & $\begin{array}{l}\text { Uses, Functions \& Services } \\
\text { rendered by Identified plant } \\
\text { species }\end{array}$ & Sampled Sites (SS) & $\begin{array}{l}\text { Control Sites } \\
\text { (CS) }\end{array}$ & $\begin{array}{l}\% \\
\text { SS }\end{array}$ & $\begin{array}{l}\% \\
\mathrm{CS}\end{array}$ \\
\hline 1 & $\begin{array}{l}\text { Environmental pollution } \\
\text { reduction \& phyto- } \\
\text { remediation }\end{array}$ & $1(18,67)$ & $4(75,78,113,115$ & 0.5 & 1.8 \\
\hline 2 & $\begin{array}{l}\text { Erosion control and } \\
\text { reclaiming of land }\end{array}$ & $5(9,10,13,14,18)$, & $6(51,67,71,108,113,114)$ & 2.3 & 2.7 \\
\hline 3 & Ornamental functions & $\begin{array}{c}24(2,7,8,9,12,17, \\
21,22,24,26,27,34,41, \\
44,46,51,55,57,61,68,6 \\
9,70,7180) \\
\end{array}$ & $7(48,51,58,66,67,73,93)$ & 11 & 3.2 \\
\hline 4 & Ethno Medicinal use & $\begin{array}{c}15(1,3,4,6,11,14 \\
20,28,31,37,50,57 \\
64,82,76)\end{array}$ & $\begin{array}{c}26(1,4,7,10,11,14,19,46,59 \\
6 \\
63,64,66,69,87,89,90,92,94 \\
, 95,106,112, \\
115,116,118,119,121)\end{array}$ & 6.9 & $\begin{array}{c}11 . \\
9\end{array}$ \\
\hline
\end{tabular}

Figures in parentheses are serial numbers given to identified plants in sampled sites and control sites

\section{DISCUSSION OF FINDINGS}

Findings showed that the identified plant species under the sampled sites are lower than that of control site with respect to species composition and individual plant species. However, the sampled sites composed of both native and non-native (exotic) plant species with more of exotic and ornamental plant species. The low plant species composition and individual plants in the sampled sites may be due to urbanization and construction. The study conducted by Mellinger et al., (2018) on diverse effect of degree of urbanization on species diversity revealed that even distribution of plant species type reduced with level of urbanization. Findings of Alexis (2013) also corroborates with these findings that the introduction of non-native plants have been on the increase in urban centers and has continued to affect plants biodiversity. Furthermore, with reference to type of identified plants more of native plants were observed in the control sites. This may have favoured species compositions because of low habitat fragmentation as urban centers are known for their high socio-economic that most timed do not consider ecosystem formations.

The degree of homogenization of plant species showed that the similarity index within sampled sites revealed index as low as $40 \%$. Thus, more non-native species were experienced in the sampled sites and this explains the degree to which more plant species are homogenized and are missing from urban greening activities. Findings are in line with Pearse et al., (2018) that urban ecosystems are widely more ecologically homogeneous than natural ecosystems and the urban 
greening practices favours plant communities that assemble from a complex mix of horticultural and regional species pools. Aronson et al., (2014) also concluded that there is strong evidence that the cultivated and spontaneous species pools in urban centers are homogenized across regions relative to the natural habitat in terms of both species and phylogenetic compositions. Groffman et al. (2014) had hypothesized that differences in plant community composition and aboveground biomass between biophysically dissimilar regions are reduced by urbanization because residential areas in different regions have more similar landscaping, and therefore plant community composition, relative to the composition of native ecosystems in these regions. Findings showed that more plant species in the control site were observed for ecosystem functions and services except ornamental functions and aesthetics and cultural diversity. These have shown that the ecological balance of the urban landscape has been affected because of the human influence in the richness and diversity of plants that are able to survive the ecological change. Alberti (2005) also reported that urban development fragments, isolates, and degrades natural habitats and as a result simplifies and homogenizes species composition; disrupts hydrological systems; and modifies energy flow and nutrient cycling. However, in general, urban landuse change has influenced on other environmental occurrences. Furthermore, Foley et al. (2005), Grimm et al. (2005) and Groffman et al. (2014) have reported that urban land-use change has been identified as one of the major components of environmental change because of its effects on climate, water, biodiversity, carbon (C), and nutrients across large areas of the globe. Groffman et al. (2014) however, stated that homogenization would be exhibited in biophysical structure, where neighbourhoods across biophysically different regions come to have similar patterns of human infrastructure (including roads, residential lots, and commercial areas), vegetation structure, and aquatic features which is being discovered in the sampled sites of this study. This homogenization may also result in ecological transformation, with replacement of natural vegetation assemblages by turfgrass, popular or weedy plant species, and impervious surfaces.

\section{CONCLUSION AND RECOMMENDATIONS}

It can be concluded that introduction of non-native plants as a result of urban greening activities have altered ecosystem functions and services. This explains why most plants grown in urban center promotes species homogenization which negative impacts will always outweigh the positive impacts. Thus, urban greening without proper consideration for habitat conservation will only encourage species homogenization and reduce ecosystem functions and services. The study recommended that urban greening projects and activities should be managed effectively by the city authorities, so as to reduce its impacts on the plants species diversity in order to improve the vegetation status and also encourage good environmental practices that will promote ecosystem functions and services.

\section{REFERENCES}

Ajewole, I.A. (2010). Urban Forestry Development in Britain and Ireland: Lessons for Nigeria. In Adeyoyoju S.K. and Bada S.O. (eds): Readings in Sustainable Tropical Forest Management. Published by Zenith Book House. pp 1-22.

Antrop M (2004) Landscape change and the urbanization process in Europe. Landscape Urban Planning 67: 9-26. 
Baiser B, Olden JD, Record S, Lockwood JL, McKinney ML (2012) Pattern and process of biotic homogenization in the New Pangaea. Proc Biol Sci 279: 4772-4777.

Chambers, D.B. (2005). Murder at Montpelier: Igbo Africans in Virginia (illustrated ed.). Univ. Press of Mississippi. p. 22.

Chowdhury, R.R. (2011). A multi-scalar approach to theorizing socio-ecological dynamics of urban residential landscapes. CATE 4(1):Article 6. Available at: http://digitalcommons.lmu.edu/cate/vol4/iss1/6.

Cohen D.M. (1994). Opisthoproctidae. In: Fishes of the North-Eastern Atlantic and the Mediterranean, Whitehead. P.J.P., M.L. Bauchot, J.C. Hureau, J. Nielsen and E. Tortonese (Eds.), UNESCO, Paris, France. (1): 395-398.

Colwell, R. K. (2009). "Biodiversity: Concepts, Patterns and Measurement". In Simon A. Levin. The Princeton Guide to Ecology. Princeton: Princeton University Press 257-263.

Conway, T.M., and Bourne, K.S. (2013). A comparison of neighborhood characteristics related to canopy cover, stem density and species richness in an urban forest. Landsc. Urban Plan, $113,10-18$.

Cooperative Forest Act (1978). Cooperative Forestry Assistance Act of 1978. Public Law 95313.http://www.house.gov/legcoun/Comps/CFAA78.PDF, 43pp.

Eludoyin, O.S., Utang P.B. and Obafemi A.A. (2012) Geographic Information Systems, Urban Forestry and Climate Change: A Review. Research Journal of Environmental and Earth Sciences 4(6): 640-645.

Eludoyin, O.S, Aiyeloja, A.A, and Ndife, O.C. (2014). Spatial Analysis of Trees Composition, Diversity and Richnesss in the Built up Areas of University of Port Harcourt, Nigeria. International Journal of Environmental and Engineering, 8(2): 142-146

Enaruvbe, G.O., and Atafo, O.P. (2015). Change in the rate and pattern of deforestation in part of the Niger Delta, Nigeria. Journal of Land use Science, 4 (1): pp 1-16

Encarta (2009). Nigeria. Microsoft. Archived from the original on 2009-11-01. Retrieved 201904-29.

Fabiyi, O.O. (2011). Change actors' analysis and vegetation loss from remote sensing data in parts of the Niger Delta region. Journal of Ecology and the Natural Environment, 3: 381391

Falola, T., and Amanda, W. (2007). Encyclopedia of the Middle Passage: Greenwood Milestones in African American History. Greenwood Publishing Group. p. 92.

Federal Republic of Nigeria (2012). 2006 Population Census" (PDF). Web.archive.org. Archived from the original (PDF) on 5 March 2012. Retrieved 2019-06-02.

Gene, W., Grey, G.W., Frederick J., and Deneke, F.J. (1978). Urban Forestry. Wiley, New York, NY. 279pp.

Geographical Alliance of Iowa, (2010). The Human and Physical Characteristics of Nigeria. University of Northern Iowa. Archived from the original on 2010-03-28. Retrieved 2019-0613.

Gilbert O.L. (1989). The ecology of urban habitats. London: Chapman and Hall. 369pp.

https://en.wikipedia.org/wiki/Species_homogeneity Retrieved 2019-05-12

Ihuma, J.O., Tella, I.O., Madakan, S.P., and Akpan, M. (2016). Frugivorous bird species diversity in relation to the diversity of fruit tree species in reserved and designated green 
areas in FCT, Nigeria. Journal of Research in Forestry, wildlife and Environment, 8 (1): 8291

Iyalla, T.M. (2001). Environmental and hydrogeological mapping: A requirement for the Niger Delta development planning. Technical paper presented to the Nigeria Society of Engineers. Technical Session in Port Harcourt, April $12^{\text {th }}$.

Kuchelmeister, G. (2000). Trees for the urban millennium: urban forestry update. Unasylva, (special issue: Trees outside forests), 51.

Kühn, I., Brandl, R., and Klotz, S. (2004). The flora of German cities is naturally species rich. Evol Ecol Res 6: 749-764.

Kühn I, and .Klotz, S. (2006) Urbanization and homogenization - comparing the floras of urban and rural areas in Germany. Biological Conservation 127: 292-300.

La Sorte, F.A., and McKinney, M.L. (2007) Compositional changes over space and time along an occurrence-abundance continuum: anthropogenic homogenization of the North American avifauna. J Biogeogr 34: 2159-2167.

Larinde, S.L., (2010) Practical Issues in Urban Forest Establishment and Management. In: Ijeoma, H.M. and A.A. Aiyeloja, (Eds.), Practical Issues in Forestry and Wildlife Resources Management, Published by Green Canopy Consultants, Choba, Port Harcourt, pp: 241-259.

Lehva“virta, S., and Rita, H., (2002). Natural regeneration of trees in urban woodlands. Journal of Vegetation Science 13, 57-66.

Lososová, Z., Chytrý, M., Tichý, L., Danihelka, J., and Fajmon K. (2012). Biotic homogenization of Central European urban floras depends on residence time of alien species and habitat types. Biological Conservation 145: 179-184.

Luc, H. and Emmanuel, K.B. (2014). Causes of Biodiversity Loss: a Human Ecological Analysis. MultiCiencia, Human Ecology Department, Belgium.

Maley J. (1996). The African rain forest-main characteristics of changes in vegetation and climate from the upper cretaceous to the quaternary. R. Soc. Edinburgh Proc. Sect. B: Biol. Sci. 104: 31-74.

McKinney, M.L., and Lockwood, J.L. (1999) Biotic homogenization: a few winners replacing many losers in the next mass extinction. Trends Ecol Evol 14: 450-453.

McKinney, M.L. (2006). Urbanization as a major cause of biotic homogenization. Biological Conservation, 11(2):247-260

McPherson, E.G., (1990). Economic modeling for large-scale urban tree plantings. In: Proceedings of the ACEEE 1990 Summer Study on Energy Efficiency in Buildings. American Council for an Energy Efficient /Economy, Washington, DC

Morrell, S.C. (2012). A Comparison of Vegetation Composition in Urban and Rural Floodplans Following Removal of Chinese Privet (Ligustrumsinense). A Master Project submitted to the Department of Geosciences, Georgia State University, USA. 65P

Oku, H. B. (2003). The Niger Delta Environment: Its Local Geography, Port Harcourt; Prelyn Publishers.

Olden, J.D., and Poff, N.L. (2003). Toward a mechanistic understanding and prediction of biotic homogenization. Am Nat 162: 442-460.

Park, C.MD. (2004). Earth Observation Researchers Seeing Double On African Monsoons. Space Media Network Promotions. Retrieved 2017-08-28. 
Pyšek, P., Bacher, S., Chytrý, M., Jarošík, V., and Wild, J. (2010) Contrasting patterns in the invasions of European terrestrial and freshwater habitats by alien plants, insects and vertebrates. Glob Ecol Biogeogr 19: 317-331.

Raupp, M., Cumming, A., and Raupp, E. (2006). Street Tree Diversity in Eastern North America and Its Potential for Tree Loss to Exotic Borers. Arboric. Urban Forestry, 2006, 32, $297-$ 304.

Rejmánek, M. (2000). A must for biogeographers. Divers Distrib 6: 208-211.

Ridley, D. (2008). The Literature Review: A step-by-step guide for students. Los Angeles: Sage.

Rindfuss, R.R., Walsh, S.J., Turner, B.L. 2nd., Fox, J., and Mishra V.(2004). Developing a science of land change: Challenges and methodological issues. Proc Natl Acad Sci USA 101(39):13976-13981.

Seto, K.C., Güneralp, B., and Hutyra, L.R. (2012). Global forecasts of urban expansion to 2030 and direct impacts on biodiversity and carbon pools. Proc Natl Acad Sci USA 109(40):16083-16088.

Simon, O.E. (2010). The Population Situation in Cross River State of Nigeria and Its Implication for Socio-Economic Development: Observations from the 1991 and 2006 Censuses" (PDF). Archived from the original (PDF) on 2 April 2012. Retrieved 2019-06-02

Sparks, R. J. (2004). The Two Princes of Calabar: An Eighteenth-century Atlantic Odyssey. Harvard University Press. p. 39.

Tan, P.; Steinbach, M.; and Kumar, V. (2005). Introduction to Data Mining, ISBN 0-321-32136$\underline{7}$

Turner, B.L., Lambin, E.F., and Reenberg, A. (2007). The emergence of land change science for global environmental change and sustainability. Proc Natl Acad Sci USA 104 (52):2066620671.

Wang, G., Jiang, G., Zhou, Y., Liu, Q., and Ji, Y. (2007) Biodiversity conservation in a fastgrowing metropolitan area in China: a case study of plant diversity in Beijing. Biodivers Conserv 16: 4025-4038.

Wear, D.N. (2013). Forecasts of Land Uses. In The Southern Forest Futures Project: Technical Report; Greis, J.G., Ed.; USDA Forest Service: Washington, DC, USA, 2013; pp. 45-72. 2.

Wood, J.P. (1999). Tree Inventories and GIS in Urban Forestry, Project report submitted to the Faculty of the Virginia Polytechnic Institute and State University in partial fulfillment of the requirements for the degree of Master in Forestry, Blacksburg, Virginia

World Bank (2010). World development indicators. World Bank publications. Washington D.C. p. 422. 